\title{
Acute electric field downregulates human plasma immunoreactive interleukin- 6 and $-1 \beta$ levels: Molecular mechanisms underlying inflammation alleviation through electric field therapy
}

\author{
Yuzo Nakagawa-Yagi ${ }^{*}$, Hiroyuki Hara ${ }^{1}$, Chisato Kanai ${ }^{2}$, Masashi Sato ${ }^{3}$ and Akikuni Hara ${ }^{1}$ \\ ${ }^{1}$ Hakuju Institute for Health Science Co. Ltd., 37-5 Tomigaya 1-chome, Shibuya-ku, Tokyo 151-0063, Japan \\ ${ }^{2}$ Kyoto Constella Technologies Co. Ltd., Kyozome Kaikan, 481 Tourouyama-cho, Nakagyo-ku, Kyoto 604-8225, Japan \\ ${ }^{3}$ Acel, Inc., SIC-2 2506, 5-4-30 Nishihashimoto, Midori-ku, Sagamihara, Kanagawa 252-0131, Japan
}

\begin{abstract}
Medical treatment using high-voltage electric potential (HELP) devices to generate an electric field (EF) is an alternative therapy commonly used in Japan. However, the mechanisms underlying potential health benefits of this therapy are still unclear. Therefore, we investigated the effect of $\mathrm{HELP}$ exposure $(9 \mathrm{kV} / \mathrm{electrode}+9 \mathrm{kV} /$ electrode, $30 \mathrm{~min}$ ) on several cytokines and hormones using enzyme-linked immunosorbent assays in plasma samples obtained from healthy human subjects before and after a single treatment session. Immunoreactive interleukin (IL)-1 $\beta$ and IL-6 levels were significantly downregulated following HELP exposure. Under these treatment conditions, HELP exposure did not exert on immunoreactive IL-10, IL-18, transforming growth factor-beta 1 (TGF- $\beta 1$ ), tumor necrosis factor-alpha (TNF- $\alpha$ ) adrenaline, serotonin, histamine, neuropeptide Y, somatostatin, insulin, or dehydroepiandrosterone sulfate (DHEAS) levels. The activation of transient receptor potential melastatin 8 (TRPM8) induces the suppression of the levels of inflammatory markers. Therefore, we further examined the in silico docking simulation of lysoPC-22:4, lysoPE-20:4, and lysoPE-22:6 with TRPM8 using a homology model. The binding energies were -10.8 , -10.4 , and -11.4 kcal/mol for lysoPC-22:4, lysoPE-20:4, and lysoPE-22:6, respectively. Our findings provide new insights into the molecular mechanisms underlying pain control and sleep quality alleviation following EF therapy.
\end{abstract}

\begin{abstract}
Abbreviations: CRP: C-reactive protein; DHEAS: dehydroepiandrosterone sulfate; EF: electric field; HELP: high-voltage electric potential; HODE: hydroxyoctadecadienoic acid; IL: interleukin; lysoPC: lysophosphatidylcholine; lysoPC-22:4: (2-\{[(2R)-3-[(7Z,10Z,13Z,16Z)docosa-7,10,13,16-tetraenoyloxy]-2-hydropropyl phosphonato]oxy\} ethyl)trimethylazanium; lysoPE: lysophosphatidylethanolamine; lysoPE-22:6: (2-aminoethoxy) [(2R)-2-[(4Z, 7Z, 10Z, 13Z, 16Z, 19Z)docosa-4,7,10,13,16-hexaenoyloxy]-3-hydroxypropoxy]phosphinic acid; lysoPE-20:4: (2-aminoethoxy) [(2R)-2-hydroxy-3-[(5Z, 8Z, 11Z, 14Z)-icosa-5,8,11,14-tetraenoyloxy] propoxy] phosphinic acid; OEA: Oleoylethanolamide; TGF- $\beta$ : transforming growth factor beta; TNF- $\alpha$ : tumor necrosis factor alpha; TRPM8: transient receptor potential melastatin 8; TRPV1: transient receptor potential vanilloid 1 .
\end{abstract}

\section{Introduction}

A therapeutic device exposing the human body to high-voltage electric potential (HELP) has been approved by the Ministry of Health, Labour and Welfare in Japan [1-19]. High-voltage electric field (EF) therapy is reported to be an effective treatment for shoulder stiffness, headache, insomnia, and chronic constipation [1-20]. Although EF therapy was discovered more than 80 years ago, the molecular mechanisms associated with its health benefits remain elusive. Altogether, the results of these studies suggest that HELP exposure may be an alternative therapy for several conditions. Our previous attempts to identify biomarker induced by HELP exposure using plasma metabolomics and lipidomics have led to the detection of lipid- derived signaling molecules such as 3-hydroxybutyrate (3-HBA), cis8,11,14-eicosatrienoic acid, 9-hydroxyoctadecadienoic acid (9-HODE), 13-hydroxyoctadecadienoic acid (13-HODE), oleoylethanolamide (OEA), lysophosphatidylethanolamine (lysoPE)-20:4, and lysoPE-22:6 [21-25]. Endogenous lipid-derived signaling molecules have been suggested as candidate molecules, representing the interface between symptoms and electroceutical target proteins [21-26]. A recent study conducted by Smani et al. reported that pretreatment with lysophosphatidylcholine (lysoPC) induced decreases in the levels of pro-inflammatory cytokines in the murine model of peritoneal sepsis caused by Acinetobacter baumannii [27]. In our previous study, we observed HELP exposure-induced upregulation of lysoPC-22:4 levels in the plasma of healthy individuals [28]. Therefore, we hypothesized that the increased plasma lysoPC-22:4 levels following EF exposure may be linked to changes in pro-inflammatory cytokines, including interleukin (IL)-1 $\beta$, IL-6, and tumor necrosis factor-alpha (TNF- $\alpha$ ). In

*Correspondence to: Yuzo Nakagawa-Yagi, $\mathrm{PhD}$, Hakuju Institute for Health Science Co. Ltd., 37-5 Tomigaya 1-chome, Shibuya-ku, Tokyo 151-0063, Japan, Tel: 81354788833, Fax: 81354789821; E-mail: yagi@hakuju.co.jp

Key words: electric field therapy, TRPM8, lysoPC-22:4, anti-inflammation, interleukin-6

Received: September 11, 2018; Accepted: September 17, 2018; Published: September 24, 2018 
Nakagawa-Yagi Y (2018) Acute electric field downregulates human plasma immunoreactive interleukin-6 and -1 $\beta$ levels: Molecular mechanisms underlying inflammation alleviation through electric field therapy

the present study, we report that the immunoreactive levels of IL-1 $\beta$ and IL- 6 may be downregulated by HELP treatment $(9 \mathrm{kV} /$ electrode +9 $\mathrm{kV} /$ electrode, $30 \mathrm{~min}$ ). In addition, we conducted a binding study to explore the interactions between lysoPC-22:4 and a homology model of transient receptor potential melastatin 8 (TRPM8) using the template structure (PDB ID 6BPQ).

\section{Materials and methods}

\section{EF exposure}

The system used for the EF exposure has been previously described [1-25,28]. The EF system was equipped with a transformer, a seat, and two insulator-covered electrodes. One electrode was placed on a floor plate on which the subject's feet were located, and the other electrode was placed above the subject's head. An EF generated by the HELP apparatus (Healthtron PRO-18T; Hakuju Institute for Health Science Co., Ltd., Tokyo, Japan) was uniformly created by transforming a 50$\mathrm{Hz}$ alternating current at $9 \mathrm{kV} /$ electrode $+9 \mathrm{kV} /$ electrode. The safety of this system for human use was confirmed by the Japanese government in 1963.

\section{Subjects}

Sixteen healthy adults ( 7 males and 9 females; mean age, $44.1 \pm$ 1.7 years; body mass index [BMI], $22.5 \pm 1.3 \mathrm{~kg} / \mathrm{m}^{2}$ ) participated in experiment 1 (exposure condition: $9 \mathrm{kV} /$ electrode $+9 \mathrm{kV} / \mathrm{electrode}, 30$ min). Twenty-five healthy adults ( 9 males and 16 females; mean age, $46.1 \pm 1.1$ years; BMI, $\left.22.2 \pm 0.5 \mathrm{~kg} / \mathrm{m}^{2}\right)$ participated in experiment 2 (exposure condition: $9 \mathrm{kV} /$ electrode $+9 \mathrm{kV} /$ electrode, $30 \mathrm{~min}$ ). All experiments were performed in the morning, and all participants signed an informed consent form after receiving verbal and written information regarding the study. All experiments were conducted in accordance with the Declaration of Helsinki, and the study protocol was approved by the human ethics committee of Hakuju Institute for Health Science Co., Ltd. (Tokyo, Japan).

\section{Plasma preparation}

Blood samples were collected using vacutainer tubes coated with ethylenediaminetetraacetic acid (VP-NA070K; Terumo Corporation, Tokyo, Japan) and immediately centrifuged at $800 \times \mathrm{g}$ for $5 \mathrm{~min}$ to separate the plasma from other cellular materials. The plasma was subsequently transferred to a fresh Eppendorf tube and stored at $-80^{\circ} \mathrm{C}$ until processing.

\section{Enzyme-linked immunosorbent assay (ELISA)}

The plasma immunoreactive levels of IL-1 $\beta$, IL-6, IL-10, IL-18, TGF- $\beta 1$, TNF- $\alpha$, adrenaline, dehydroepiandrosterone sulfate (DHEAS), histamine, insulin, neuropeptide $\mathrm{Y}$, serotonin, and somatostatin were measured using a human IL- $1 \beta$, IL-6, IL-10, TGF- $\beta 1$ or TNF- $\alpha$ ELISA kit (R\&D systems, Minneapolis, MN, USA), a human IL-18 ELISA kit (Medical \& Biological Laboratories, Nagoya, Japan), a human adrenaline and DHEAS ELISA kit (Lifespan Biosciences, Seattle, WA, USA), a human histamine ELISA kit (Bertin Pharma, Montigny le Bretonneux, France), a human insulin ELISA kit (Mercodia, Uppsala, Sweden), a human neuropeptide Y ELISA kit (Cloud-Clone, Houston, TX, USA), a human serotonin ELISA kit (Enzo Life Sciences, Farmingdale, NY, USA), or a human somatostatin ELISA kit (RayBiotech, Norcross, GA, USA).

\section{Homology modeling and docking simulation}

We used Q7Z2W7.fasta registered in UniProt to obtain the sequence of human TRPM8 (hTRPM8). The three-dimentional structure (6BPQ;
Protein Data Bank Japan) was used as a template structure. The best docking score was chosen from the docking calculation between icilin (an agonist of TRPM8) and a homology model. The docking study of lysoPC-22:4 binding to the target protein of the hTRPM8 model structure was performed using the AutoDock Vina docking software (Dr. Oleg Trott, The Scripps Research Institute, CA, USA) [29]. The docking experiment was performed five times and yielded 100 candidate conformations.

\section{Statistical analysis}

Data were analyzed using Welch's $t$-test. A probability $(p)$ value $<$ 0.05 was considered statistically significant.

\section{Results}

\section{Effect of HELP exposure on immunoreactive cytokines in the plasma of healthy individuals}

We examined the effect of HELP exposure $(9 \mathrm{kV} /$ electrode $+9 \mathrm{kV} /$ electrode) for $30 \mathrm{~min}$ on immunoreactive cytokines in the plasma of healthy individuals at multiple time points (Figure 1). The levels of immunoreactive IL- $1 \beta$ were significantly downregulated at the 0time and 30-min time point after HELP exposure compared with those observed before the exposure (IL- $1 \beta$-After 0time: 0.66 -fold, $p=0.00005$; IL-1 $\beta$-After 30-min: 0.83-fold, $p=0.025$ ). Moreover, the levels of immunoreactive IL- 6 were significantly downregulated at the 30 -min time point after HELP exposure compared with those observed before the exposure (IL-6: 0.68-fold, $p=0.039$ ). Under these conditions, HELP exposure did not affect the levels of immunoreactive IL-10, IL-18, TNF- $\alpha$, or TGF- $\beta 1$ (Figure 1c-f).

\section{Effect of HELP exposure on immunoreactive hormones in the plasma of healthy individuals}

We examined the effect of HELP exposure $(9 \mathrm{kV} /$ electrode $+9 \mathrm{kV} /$ electrode) for $30 \mathrm{~min}$ on immunoreactive hormones in the plasma of healthy individuals to determine the specificity (Table 1). HELP exposure did not affect the levels of immunoreactive adrenaline, DHEAS, histamine, insulin, neuropeptide $Y$, serotonin, or somatostatin.

Docking simulation of lysoPC-22:4, lysoPE-20:4, and lysoPE-22:6 with a homology model of TRPM8

Acute EF exposure induces a notable increase in the levels of lysoPC-22:4 in the plasma of healthy subjects [28]. LysoPC-16:0 activates TRPM8 in CHO cells expressing TRPM8 [30]. Therefore, we examined the in silico docking of lysoPC-22:4 in the active site of TRPM8 using the AutoDock Vina software. We set the number of outputs poses to 20 , with a total of 100 candidate conformations. LysoPC-22:4 showed good binding energy $(-10.8 \mathrm{kcal} / \mathrm{mol})$ (Table 2). LysoPC-22:4 formed hydrogen bonds with Tyr-745, Glu-782, and Tyr-1005 (Figure 1a). The results indicate that lysoPC-22:4 binds to the TRPM8 channel. Under these conditions, icilin (a well-known TRPM8 agonist) showed a strong interaction energy of $-11.4 \mathrm{kcal} / \mathrm{mol}$ (Figure $1 \mathrm{~b}$, Table 2). In addition, we examined the in silico docking of lysoPE-20:4 and lysoPE-22:6 to the active site of TRPM8. A similar docking score was obtained using lysoPE-20:4 instead of lysoPC-22:4 (Table 2). LysoPE-22:6 showed a strong interaction energy of $-11.4 \mathrm{kcal} / \mathrm{mol}$ (Table 2). LysoPE-20:4 interacted with Glu-782, Asn-799, Asp-802, Arg-842, and Tyr-1005, and lysoPE-22:6 interacted with Asn-799 and Arg-842 (Figures 1c and 1d, Table 2). Subsequently, we examined the in silico docking of 13HODE, 9-HODE, and OEA to the active site of TRPM8 to determine 
Nakagawa-Yagi Y (2018) Acute electric field downregulates human plasma immunoreactive interleukin-6 and -1 levels: Molecular mechanisms underlying inflammation alleviation through electric field therapy
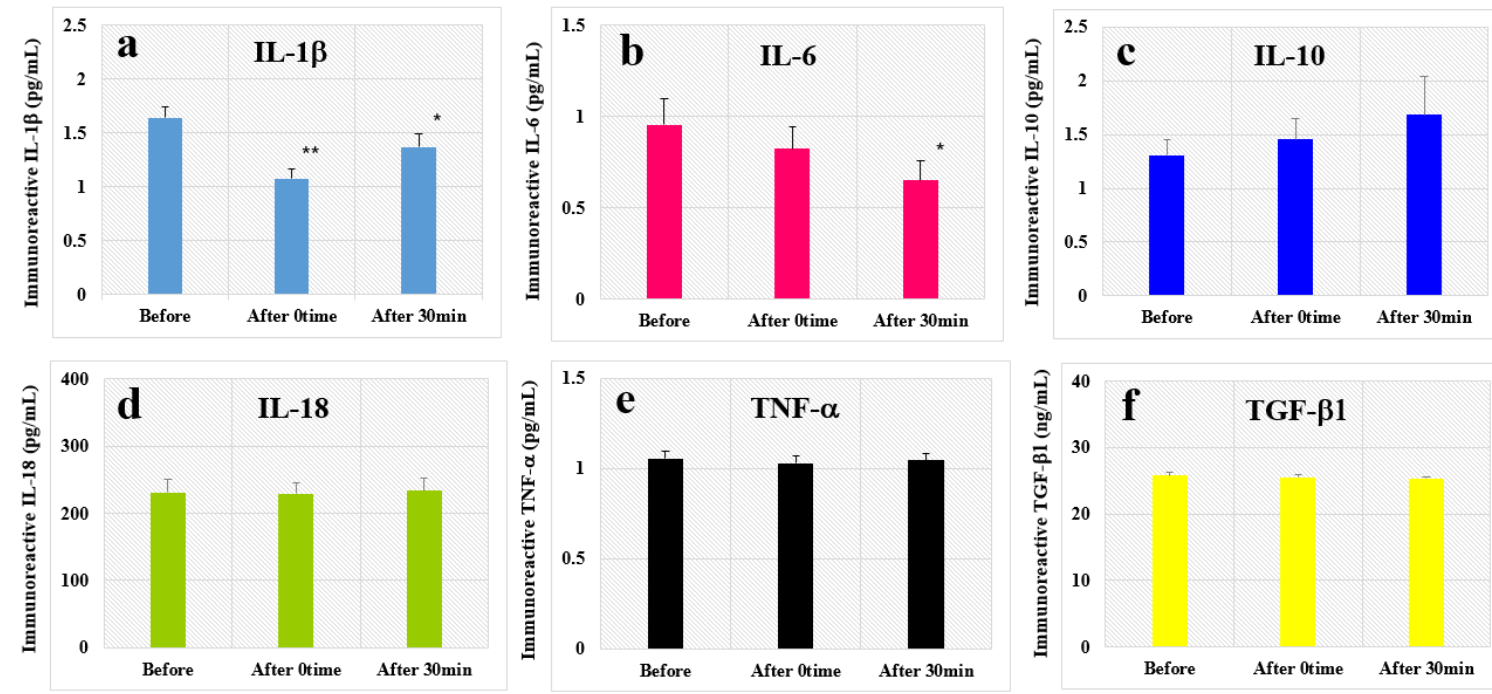

Figure 1. Effect of HELP exposure on immunoreactive cytokine levels in the plasma of healthy individuals

Effect of HELP exposure ( 9 kV/electrode $+9 \mathrm{kV} /$ electrode, $30 \mathrm{~min})$ exposure on the plasma levels of immunoreactive IL-1 $\beta$ (a), IL-6 (b), IL-10 (c), IL-18 (d), TNF- $\alpha$ (e), and TGF- $\beta 1$ (f) at multiple time points. ${ }^{*} p<0.05$ compared with before exposure. ${ }^{* *} p<0.01$ compared with before exposure.
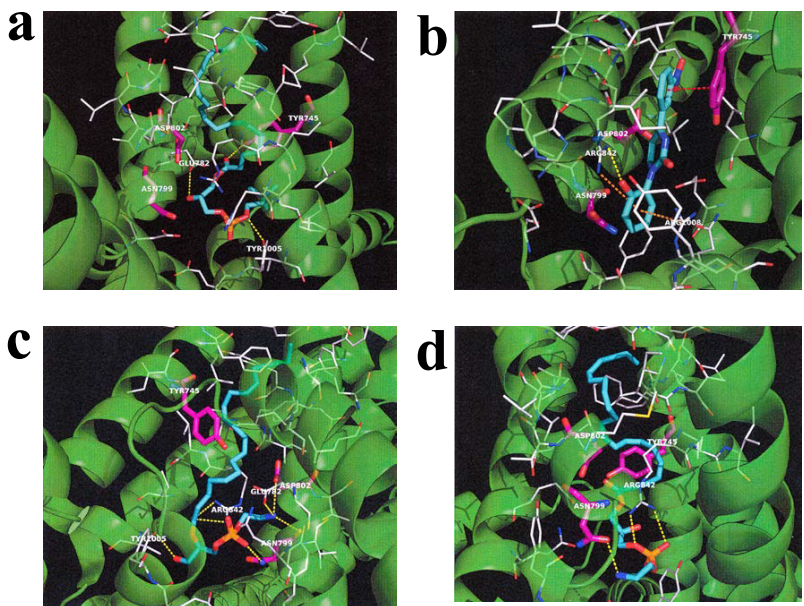

Figure 2. In silico molecular docking of lysoPC-22:4, icilin, lysoPE-20:4, and lysoPE-22:6 in the TRPM8 active site

(a) Binding mode of lysoPC-22:4 in the homology modeling of TRPM8. The yellow dashed line indicates hydrogen bonding. The carboxyl of the ester moiety of lysoPC-22:4 forms a hydrogen bond with the hydroxyl group of Tyr-745. The hydroxyl group of lysoPC-22:4 forms a hydrogen bond with the carboxyl group of Glu-782. The phosphoryl ester moiety of lysoPC-22:4 forms a hydrogen bond with the hydroxyl group of Tyr-1005. (b) Binding mode of icilin in the homology modeling of TRPM8. The yellow dashed line indicates hydrogen bonding. The hydroxyl group of icilin forms a hydrogen bond with the guanidyl group of Arg-842. The red dashed line indicates $\pi-\pi$ interaction. The nitrobenzene of icilin and the benzene ring of Tyr- 745 interact with a $\pi-\pi$. The orange dashed line indicates a cation- $\pi$ interaction. The phenol moiety of icilin and the guanidyl group of Arg- 842 or Arg- 1008 interact with cation- $\pi$. (c) Binding mode of lysoPE-20:4 in the homology modeling of TRPM8. The yellow dashed line indicates hydrogen bonding. The carboxyl of the ester moiety of lysoPE-20:4 forms a hydrogen bond with the guanidyl group of Arg-842. The phosphonyl group of lysoPE-20:4 forms a hydrogen bond with the carboxamide group of Asn-799. The primary amine group of lysoPE-20:4 forms a hydrogen bond with the carboxyl group of Glu-782 or Asp-802. The primary amine group of lysoPE-20:4 forms a hydrogen bond with the carboxamide group of Asn799. The hydroxyl group of lysoPE-20:4 forms a hydrogen bond with the hydroxyl group of Tyr-1005. (d) Binding mode of lysoPE-22:6 in the homology modeling of TRPM8. The yellow dashed line indicates hydrogen bonding. The phosphoryl ester moiety of lysoPE-22:6 forms a hydrogen bond with the guanidyl group of Arg-842. The phosphonyl group of lysoPE-22:6 forms a hydrogen bond with the guanidyl group of Arg- 842 . The quaternary amine group of lysoPE-22:6 forms a hydrogen bond with the carboxamide group of Asn-799.

Table 1. Effect of HELP exposure $(9 \mathrm{kV} /$ electrode $+9 \mathrm{kV} / \mathrm{electrode}, 30 \mathrm{~min})$ on immunoreactive hormone levels in the plasma of healthy individuals

\begin{tabular}{|c|c|c|c|c|}
\hline & & Before & After & Ratio \\
\hline & $\mathbf{n}$ & Mean \pm SE & Mean \pm SE & After/Before \\
\hline Adrenaline $(\mathrm{ng} / \mathrm{mL})$ & 16 & $20.9 \pm 0.9$ & $20.5 \pm 0.9$ & 0.98 \\
\hline DHEAS $(\mathrm{ng} / \mathrm{mL})$ & 16 & $144 \pm 27$ & $139 \pm 20$ & 0.97 \\
\hline Histamine $(\mathrm{nM})$ & 25 & $1.16 \pm 0.37$ & $1.26 \pm 0.38$ & 1.09 \\
\hline Insulin $(\mathrm{mU} / \mathrm{L})$ & 16 & $12.2 \pm 2.6$ & $10.5 \pm 2.0$ & 0.86 \\
\hline Neuropeptide $\mathrm{Y}(\mathrm{pg} / \mathrm{mL})$ & 25 & $124 \pm 5$ & $117 \pm 6$ & 0.94 \\
\hline Serotonin $(\mu / \mathrm{mL})$ & 25 & $1.09 \pm 0.08$ & $1.19 \pm 0.09$ & 0.50 \\
\hline Somatostatin $(\mathrm{ng} / \mathrm{mL})$ & 25 & $52.1 \pm 3.5$ & $49.2 \pm 3.2$ & 0.170 \\
\hline
\end{tabular}


Nakagawa-Yagi Y (2018) Acute electric field downregulates human plasma immunoreactive interleukin-6 and -1 $\beta$ levels: Molecular mechanisms underlying inflammation alleviation through electric field therapy

Table 2. Docking score and key interacting residues of TRPM8

\begin{tabular}{|c|c|c|}
\hline Ligand & Docking score (kcal/mol) & Interactive residues \\
\hline Icilin & -11.4 & Tyr-745, Arg-842, and Arg-1008 \\
\hline LysoPC-22:4 & -10.8 & Tyr-745, Glu-782, and Tyr-1005 \\
\hline LysoPE-20:4 & -10.4 & Glu-782, Asn-799, Asp-802, Arg-842, and Tyr-1005 \\
\hline LysoPE-22:6 & -11.4 & Asn-799, and Arg-842 \\
\hline 13-HODE & -9.1 & Asp-802 \\
\hline 9-HODE & -9.3 & \\
\hline OEA & -9.8 & Asp-781 \\
\hline
\end{tabular}

the specificity. The binding energies were $-9.1,-9.3$, and $-9.8 \mathrm{kcal} / \mathrm{mol}$ for 13-HODE, 9-HODE, and OEA, respectively (Table 2).

\section{Discussion}

In this study, we showed that the levels of IL-1 $\beta$ and IL- 6 are sensitive to acute EF exposure in healthy humans. Notably, the absence of a pro-inflammatory cytokine TNF- $\alpha$ response indicates that the IL- $1 \beta$ and IL- 6 responses are not adverse nonspecific actions of the immune function in humans. Our previous studies have shown that the IL-6 levels in the plasma of healthy individuals are ineffective after 15 min of acute EF exposure [22]. An acute EF exposure of 30 min may be necessary to develop a significant inhibitory effect on the IL-6 levels in the plasma. Further studies are warranted to identify the optimal condition for the downregulation of pro-inflammatory cytokines such as IL- 6 and IL- $1 \beta$ induced by EF exposure. The molecular mechanisms of changes in the plasma levels of IL- 6 and IL- $1 \beta$ following HELP exposure are complex and may be interpreted in several ways. The endogenous lipid-derived metabolite 3-hydroxybutyrate (3-HBA) has been suggested to function as an endogenous inhibitor of the NLRP3 inflammasome [31]. Using nontargeted human metabolomics, we recently demonstrated that the increase in plasma 3-HBA levels is elicited by EF exposure [24]. Interestingly, Youm et al. reported that 3 -HBA inhibits the secretion of IL-1 $\beta$ in LPS-stimulated human monocytes without significantly affecting the production of TNF- $\alpha$ [32]. In addition, there is evidence that IL-6 is a known downstream target of IL- $1 \beta$, consistently increased in the blood of patients with NLRP3 inflammasome-mediated conditions [33]. Thus, it is reasonable to speculate that EF exposure may inhibit the NLRP3 inflammasome through the upregulation of 3-HBA.

Furthermore, we previously showed an acute EF exposure $(9 \mathrm{kV} /$ electrode $+9 \mathrm{kV} /$ electrode, $30 \mathrm{~min}$ )-induced increase in the levels of lysoPC-22:4 (approximately 1.47-fold) in other lipid-derived signaling molecules [28]. Considering the role of the TRP channel family in changes in the plasma levels of lysoPC-22:4, Andersson et al. reported an increase in $\left[\mathrm{Ca}^{2+}\right]$ induced by lysoPC-16:0 in CHO cells transfected with TRPM8 [30]. Unfortunately, lysoPC-22:4 is not commercially available as a pure chemical reagent for pharmacological experiments. Thus, at present, it is not possible to investigate the effect of lysoPC-22:4 on the intracellular levels of calcium in CHO-K1 or HEK293T cells stably expressing hTRPM8. An increasing number of reports on virtual simulation are available in the literature [25,28,34]. Studies involving in silico molecular docking have been conducted to support the pharmacological results. However, the crystal structure of hTRPM8 has not yet been determined. Thus, we focused on the homology modeling of TRPM8. In the present study, the docking simulation showed that lysoPC-22:4 has good binding affinity $(-10.8 \mathrm{kcal} / \mathrm{mol})$. The docking scores were compared with several well-known transient receptor potential vanilloid 1 (TRPV1) agonists such as 13-HODE, 9-HODE, and OEA to determine the relative affinity further $[35,36]$. These results suggest that TRPV1 agonists exhibit weaker affinity than icilin,
lysoPC-22:4, lysoPE-20:4, and lysoPE-22:6 with a homology model of TRPM8. In key interacting residues, a previous study using the TRPM8 homology model (PDB ID: 1QGR) with icilin binding pockets reported hydrogen bonding to Tyr-745 [37]. Another study on menthol, a wellknown TRPM8 agonist, showed that mutating arginine at position 842 in S4 of TRPM8 to alanine decreases the affinity for menthol [38]. Once the crystal structure of TRPM8 is determined, it may be interesting to identify the binding pocket of lysoPC-22:4, lysoPE-20:4, and lysoPE-22:6 in hTRPM8. However, lysoPC-22:4, lysoPE-20:4, and lysoPE-22:6 also activate the $\mathrm{G}$ protein-coupled receptor 119 , raising the possibility that these receptors may serve as targets for lysoPC-22:4, lysoPE-20:4, and lysoPE-22:6 during EF exposure [25].

Considerable evidence for the modulation of cytokines has been obtained from animal models of arthritis and neuropathic pain [3942]. In particular, Naito et al. reported that static EF exposure inhibits the increased expression of IL1 $\beta$, but not of TNFa in arthitic hind paws [40]. Interestingly, the sensitivity of cytokines in that study was comparable to that observed in the present study. On the other hand, Khalil et al. reported that TRPM8 in macrophages modulates colitis through a balance-shift in the production of pro-inflammatory and antiinflammatory cytokines [43]. Moreover, Ramachandran et al. reported that activation of TRPM8 by icilin attenuates trinitrobenzenesulfonic acid- or dextran sodium sulfate-induced colonic inflammation in in vivo models [44]. Thus, it is reasonable to speculate that EF exposure may alleviate inflammation through the binding of TRPM8 by lysoPC-22:4, lysoPE-20:4, and lysoPE-22:6. However, it is unclear whether changes in the levels of IL-1 $\beta$ and IL- 6 may be attributed to neurons, macrophages, melanocytes, or keratinocytes. Although the underlying mechanisms of anti-inflammation by EF exposure remain to be elucidated, the role of lysoPC-22:4, lysoPE-20:4, or lysoPE-22:6 as endogenous agonist of TRPM8 may be potential mechanisms. Thus, it is conceivable that the decrease in IL- $1 \beta$ and IL- 6 levels is, at least in part, responsible for the improvement observed in leprosy patients with neuroinflammation undergoing EF exposure [2-3]. Moreover, Proudfoot et al. reported that activation of TRPM8 elicits analgesia in chronic neuropathic pain models [45]. There is also evidence that activation of TRPM8 exerts an analgesic effect on acute and inflammatory pain [46]. Interestingly, Vanmolkot and de Hoon reported increased blood C-reactive protein (CRP) levels in young adult patients with migraine [47]. Using lipidomic analysis of serum samples, Ren et al. recently reported that the levels of lysoPE-22:6 are decreased in migraine patients [48]. Thus, it is reasonable to speculate that EF exposure alleviates headache such as migraine via upregulation of lysoPE-22:6. It may be interesting to evaluate the possible effect of EF exposure on migraine in future studies.

Chronic inflammation in aging has also been proposed as a strong risk factor for morbidity and mortality in elderly individuals [49]. Of note, centenarians cope with chronic subclinical inflammation through an anti-inflammatory response termed "anti-inflammaging" [50]. It 
Nakagawa-Yagi Y (2018) Acute electric field downregulates human plasma immunoreactive interleukin-6 and -1 $1 \beta$ levels: Molecular mechanisms underlying inflammation alleviation through electric field therapy

may be interesting to evaluate the possible effect of repetitive HELP exposure on human longevity in future studies.

Considerable evidence regarding an association between IL-6 and sleep quality has been obtained from studies involving aging females and a meta-analysis of cohort studies [51-53]. Interestingly, Irwin et al. reported that sleep disturbance is associated with high levels of CRP and IL-6, but not TNF- $\alpha$ [54]. In contrast, Milrad et al. reported that poor sleep quality is associated with greater circulating levels of TNF- $\alpha$, IL- $1 \beta$, and IL-6 [55]. In the present study, repeated EF treatment was not performed. Therefore, it is reasonable to speculate that EF therapy alleviates insomnia, at least in part, through the downregulation of IL$1 \beta$ and IL- $6[6,15,16,20]$. Further basic research studies are warranted to elucidate the alleviative effect of endogenous lipid-derived signaling molecules such as lysoPC-22:4, lysoPE-20:4, or lysoPE-22:6 on sleep disturbance.

In conclusion, acute HELP exposure induced marked inhibitory effects on the plasma levels of IL- $1 \beta$ and IL-6 in healthy individuals. In silico molecular docking of lysoPC-22:4, lysoPE-20:4, and lysoPE-22:6 was observed for TRPM8. Our findings provide insight into the molecular mechanisms involved in the alleviation of headache and insomnia induced by the HELP device. These mechanisms may also be important for defense against inflammaging.

\section{Competing interests}

YN-Y, HH, and AH are employed by Hakuju Institute for Health Science Co., Ltd.; CK is employed by Kyoto Constella Technologies Co., Ltd.; and MS is employed by Acel Inc. The remaining authors have no competing interests.

\section{Authors' contributions}

YN-Y designed and supervised the research and wrote the manuscript. YN-Y, HH, AH, and MS performed EF exposure and ELISA experiments. CK performed molecular modeling. All authors have read and approved the final version of the manuscript.

\section{Acknowledgment}

We thank Dr. Makoto Kikuchi (Professor Emeritus, National Defense Medical College, Japan) for encouragement. We thank Katherine Nixon who provided medical writing services on behalf of Enago Crimson Interactive Pvt. Ltd.

\section{References}

1. Hara H (1961) On the effect of AC. electrostatic high voltage potential load upon the blood-electrolytes. Niigata Medical J 75: 265-273.

2. Shiga K, Nakamura K, Kasuga T, Tomoda M, Matsuo T (1967) AC high-voltage electrostatic neuralgia therapy. Iryo 21: 318 .

3. Nakamura K, Minami S (1970) Effect of high-voltage electrostatic therapy on neuropathic pain in leprosy. Iryo 24: 328-329.

4. Ito F, Furuya K (1981) The effect of high voltage alternating current upon a human body the change of blood pressure, endocrine system and serum lipids. $J \mathrm{Jpn} \mathrm{Sci}$ Balneol Climatol Phys Med 45: 6-17.

5. Isaka K, Nishimura R, Arase S, Takiwaki H, Osaki K, et al. (1998) Dosimetry and exposure experiments for extremely low frequency high-tension electric field therapy. EMC '98 Rome International Symposium on Electromagnetic Comoatibility D: 204207

6. Nawarat S, Iomsai K, Jantanam P, Kauengtip Y (1999) Effects of electrical Healthtron on curing of non-communicable diseases: Case study of Banlad hospital Petchaburi province. Region 4 Medical J 18: 139-149.
7. Ito F (2000) The role of electric field therapeutic device (Healthtron) in the therapy of acute low back pain. J Jpn Sci Balneol Climatol Phys Med 63: 127-137.

8. Siripanichgon K, Otrakul A, Suparp J, Sirikulchayanonta C, Charupoonphol P (2000) Clinical observation of Healthtron therapy. J Public Health (Bangkok) 30: 19-29.

9. Sirikulchayanonta C, Siripanichgon K, Otrakul A, Suparp J, Charupoonphol P (2001) The effect of Healthtron on serum lipid levels among the middle-aged: Preliminary report. J Public Health (Bangkok) 31: 63-70.

10. Sung KK, Hwang CY, Lee SK, Lee SY, Cheong SS, et al. (2002) The clinical effect of high voltage AC field therapy (Healthtron) on peripheral circulatory disturbance and functional outcome of rehabilitation in CVA patients. Korean J Oriental Physiol Pathol 16: 609-615.

11. Ito F, Ohsaki K, Takahashi K, Hara H (2005) The effects of electric field therapeutic device (Healthtron) on the stiffness in the neck and shoulder area - changes in subjective symptoms, blood circulation and the autonomic nervous system. J Jpn Sci Balneol Climatol Phys Med 68: 110-121.

12. Hua G, Lin S, Wang Y, Yin F, Shi W, et al. (2006) The effects and safety of the electric field of a high voltage alternating current for menopausal women. Chin J Phys Med Rehabil 28: 337-340.

13. Onigbinde AT, Adedoyin RA (2010) Effect of high voltage Healthtron device on blood pressure and plasma glucose of normal subjects - A technical report. Indian J Physiother Occup Ther 4: 29-31.

14. Owoeye O, Temiye E (2010) Therapeutic efficacy of attenuated high voltage Healthtron device on children with cerebral palsy. Nigerian J Medical Rehabilitation 15: 17-22.

15. Zhang L, Wang Q, Peng N, Chen W, Li X, et al. (2012) High-voltage electrostatic therapy for chronic sleep disorder in aged patients. J Chinese PLA Postgrad Med Sch 33: $730-732$.

16. Zhang L, Peng N, Chen W, Wang Q, Li X, et al. (2012) Effect of high-voltage electrostatic therapy on sleep disorder in older adults. Chin J Rehabil Theory Pract 18: 286-288.

17. Shinba T, Takahashi K, Kanetaka S, Nedachi T, Yamaneki M, et al. (2012) A pilot study on electric field therapy for chronic pain with no obvious underlying diseases. Soc Integrative Med Jpn 5: 68-72.

18. Yanamoto H, Nakajo Y, Kataoka H, Iihara K (2013) High voltage electric potentials to enhance brain-derived neurotrophic factor levels in the brain. Front Neurol Neurosci 32: 129-138. [Crossref]

19. Mitani Y, Matsugi A, Okano H, Nedachi T, Hara H (2015) Effect of exposure to a high-voltage alternating current electric field on muscle extensibility. J Jpn Sci Balneol Climatol Phys Med 78: 244-252.

20. Ohtsuki T, Nabeta T, Nakanishi H, Kawahata H, Ogihara T, et al. (2017) Electric field exposure improves subjective symptoms related to sleeplessness in college students: A pilot study of electric field therapy for sleep disorder. Curr Med Chem 17: 37-48. [Crossref]

21. Nakagawa-Yagi Y, Hara H, Fujimori T, Yamaguchi T, Midorikawa A, et al. (2014) Non-targeted human plasma metabolomics reveals the changes in oleoylethanolamide, a lipid-derived signaling molecule, by acute exposure of electric field. Integr $\mathrm{Mol} \mathrm{Med}$ 1: $29-37$

22. Nakagawa-Yagi Y, Hara H, Yoshida Y, Midorikawa A, Hara A (2015) Discovery of a novel effect of electric field exposure on human plasma beta-endorphin and interleukin-12 levels: Insight into mechanisms of pain alleviation and defence against infection by electric field therapy. Integr Mol Med 2: 200-204.

23. Nakagawa-Yagi Y, Hara H, Nakagawa F, Sato M, Hara A (2016) Acute exposure to an electric field induces changes in human plasma 9-HODE, 13-HODE, and immunoreactive substance P levels: Insight into the molecular mechanisms of electric field therapy. Integr Mol Med 3: 600-605.

24. Nakagawa-Yagi Y, Hara H, Tsuboi H, Abe J, Hara A(2016) Effect of 3-hydroxybutyrate, an endogenous histone deacetylase inhibitor, on FOXO3A mRNA expression in human epithelial colorectal Caco-2 cells: Insight into the epigenetic mechanisms of electric field therapy. Integr Mol Med 3: 764-768.

25. Nakagawa-Yagi Y, Hara H, Nakanishi H, Kanai C, Hara A (2017) Molecular insight into the docking of lysophosphatidylethanolamine (lysoPE)-22:6 interaction with GPR119: Acute exposure to an electric field induces changes in human plasma lysoPE-22:6 and lysoPE-20:4 levels. Integr Mol Med 4: 1-7.

26. Taberner FJ, Fernandez-Ballester G, Fernandez-Carvajal A, Ferrer-Montiel A (2015) TRP channels interaction with lipids and its implications in disease. Biochim Biophys Acta 1848: 1818-1827. [Crossref] 
Nakagawa-Yagi Y (2018) Acute electric field downregulates human plasma immunoreactive interleukin-6 and -1 levels: Molecular mechanisms underlying inflammation alleviation through electric field therapy

27. Smani Y, Dominguez-Herrera J, Ibanez-Martinez J, Pachon J (2015) Therapeutic efficacy of lysophosphatidylcholine in severe infections caused by Acinetobacter baumannii. Antimicrobial Agents and Chemotherapy 59: 3920-3924.

28. Nakagawa-Yagi Y, Hara H, Nakanishi H, Tasaka T, Hara A (2017) Acute exposure to an electric field induces changes in human plasma lysophosphatidylcholine (lysoPC)-22:4 levels: Molecular insight into the docking of lysoPC-22:4 interaction with TRPV2. Integr Mol Med 4: 1-7.

29. Trott O, Olson AJ (2010) AutoDock Vina: improving the speed and accuracy of docking with a new scoring function, efficient optimization, and multithreading. $J$ Comput Chem 31: 455-461. [Crossref]

30. Andersson DA, Nash M, Bevan S (2007) Modulation of the cold-activated channe TRPM8 by lysophospholipids and polyunsaturated fatty acids. J Neurosci 27: 3347 3355. [Crossref]

31. Goldberg EL, Asher JL, Molony RD, Shaw AC, Zeiss CJ, et al. (2017) $\hat{\mathrm{I}}^{2}$ Hydroxybutyrate Deactivates Neutrophil NLRP3 Inflammasome to Relieve Gout Flares. Cell Rep 18: 2077-2087. [Crossref]

32. Youm Y-H, Nguyen KY, Grant RW, Goldberg EL, Bodogai M, et al. (2015) Ketone metabolite $\beta$-hydroxybutyrate blocks NLRP3 inflammasome-mediated inflammatory disease. Nat Med 21: 263-269.

33. Ridker PM (2016) From C-Reactive Protein to Interleukin-6 to Interleukin-1: Moving Upstream To Identify Novel Targets for Atheroprotection. Circ Res 118: 145-156. [Crossref]

34. Nakagawa-Yagi Y, Sato Y, Matsumoto E, Nakatsuka S, Sakaki T, et al. (2012) Pharmacological modulation of histone demethylase activity by a small molecule isolated from subcritical water extracts of Sasa senanensis leaves prolongs the lifespan of Drosophila melanogaster. BMC Complement Altern Med 12: 101. [Crossref]

35. Ahern GP (2003) Activation of TRPV1 by the satiety factor oleoylethanolamide. J Bio Chem 278: 30429-30434. [Crossref]

36. Patwardhan AM, Akopian AN, Ruparel NB, Diogenes A, Weintraub ST, et al. (2010) Heat generates oxidized linoleic acid metabolites that activate TRPV1 and produce pain in rodents. J Clin Invest 120: 1617-1626. [Crossref]

37. Pedretti A, Marconi C, Bettinelli I, Vistoli G (2009) Comparative modeling of the quaternary structure for the human TRPM8 channel and analysis of its features. Biochim Biophys Acta 1788: 973-982. [Crossref]

38. Janssens A, Voets T (2011) Ligand stoichiometry of the cold- and menthol-activated channel TRPM8. J Physiol 589: 4827-4835. [Crossref]

39. Pan RY, Chen SL, Xiao X, Liu DW, Peng HJ, et al. (2000) Therapy and prevention of arthriris by recombinant adeno-associated virus vector with delivery of interleukin-1 receptor antagonist. Arthritis Rheum 43: 289-297. [Crossref]

40. Naito Y, Aoi W, Inoue M, Takagi T, Akagiri S, et al. (2009) Static electric field by high voltage alternating current ameliorates collagen-induced arthritis in mice via the inhibition of IL-1 $\beta$ expression. J Complement Integr Med 6: 30 .

41. Sacerdote P, Franchi S, Moretti S, Castelli M, Procacci P, et al. (2013) Cytokine modulation is necessary for efficacious treatment of experimental neuropathic pain. $J$ Neuroimmune Pharmacol 8: 202-211.
42. Venkatesha SH, Dudics S, Acharya B, Moudgil KD (2015) Cytokine-modulating strategies and newer cytokine targets for arthritis therapy. Int J Mol Sci 16: 887-906. [Crossref]

43. Khalil M, Babes A, Lakra R, Försch S, Reeh PW, et al. (2016) Transient receptor potential melastatin 8 ion channel in macrophages modulates colitis through a balanceshift in TNF-alpha and interleukin-10 production. Mucosal Immunol 9: 1500-1513. [Crossref]

44. Ramachandran R, Hyun E, Zhao L, Lapointe TK, Chapman K, et al. (2013) TRPM8 activation attenuates inflammatory responses in mouse models of colitis. Proc Natl Acad Sci USA 110: 7476-7481. [Crossref]

45. Proudfoot CJ, Garry EM, Cottrell DF, Rosie R, Anderson H, et al. (2006) Analgesia mediated by the TRPM8 cold receptor in chronic neuropathic pain. Curr Biol 16: 15911605. [Crossref]

46. Liu B, Fan L, Balakrishna S, Sui A, Morris JB, et al. (2013) TRPM8 is the principa mediator of menthol-induced analgesia of acute and inflammatory pain. Pain 154 2169-2177. [Crossref]

47. Vanmolkot FH, de Hoon JN (2007) Increased C-reactive protein in young adult patients with migraine. Cephalalgia 27: 843-846. [Crossref]

48. Ren C, Liu J, Zhou J, Liang H, Wang Y, et al. (2018) Lipidomic analysis of serum samples from migraine patients. Lipids Health Dis 17: 22. [Crossref]

49. Franceschi C, Campisi J (2014) Chronic inflammation (inflammaging) and its potentia contribution to age-associated diseases. J Gerontol A Biol Sci Med Sci 69: S4-S9. [Crossref]

50. Minciullo PL, Catalano A, Mandraffino G, Casciaro M, Crucitti A, et al. (2016) Inflammaging and Anti-Inflammaging: The Role of Cytokines in Extreme Longevity. Arch Immunol Ther Exp (Warsz) 64: 111-126. [Crossref]

51. Friedman EM, Hayney MS, Love GD, Urry HL, Rosenkranz MA, et al. (2005) Social relationships, sleep quality, and interleukin-6 in aging women. Proc Natl Acad Sci USA 102: 18757-18762. [Crossref]

52. Hong S, Mills PJ, Loredo JS, Adler KA, Dimsdale JE (2005) The association between interleukin-6, sleep, and demographic characteristics. Brain Behav Immun 19: 165-172. [Crossref]

53. Okun ML, Hall M, Coussons-Read MR (2007) Sleep disturbances increase interleukin-6 production during pregnancy: Implications for pregnancy complications. Reprod Sci 14: 560-567. [Crossref]

54. Irwin MR, Olmstead R, Carroll JE (2016) Sleep Disturbance, Sleep Duration, and Inflammation: A Systematic Review and Meta-Analysis of Cohort Studies and Experimental Sleep Deprivation. Biol Psychiatry 80: 40-52. [Crossref]

55. Milrad SF, Hall DL, Jutagir DR, Lattie EG, Ironson GH, et al. (2017) Poor sleep quality is associated with greater circulating pro-inflammatory cytokines and severity and frequency of chronic fatigue syndrome/myalgic encephalomyelitis (CFS/ME) symptoms in women. J Neuroimmunol 303: 43-50. [Crossref]

Copyright: (C2018 Nakagawa-Yagi Y. This is an open-access article distributed under the terms of the Creative Commons Attribution License, which permits unrestricted use, distribution, and reproduction in any medium, provided the original author and source are credited. 